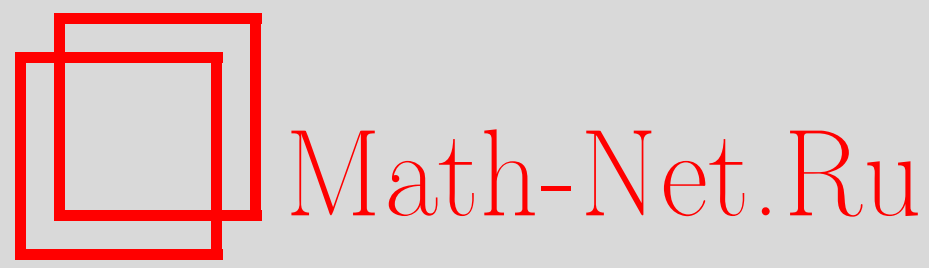

В. Ю. Протасов, К вопросу об алгоритмах приближенного вычисления минимума выпуклой функции по ее значениям, Матем. заметки, 1996, том 59, выпуск 1, 95-102

DOI: https://doi.org/10.4213/mzm1697

Использование Общероссийского математического портала MathNet.Ru подразумевает, что вы прочитали и согласны с пользовательским соглашением

http://www . mathnet.ru/rus/agreement

Параметры загрузки:

IP : 54.166 .219 .16

26 апреля 2023 г., $17: 59: 27$

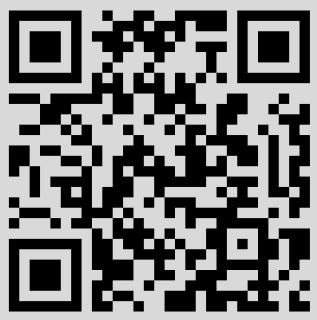




\section{К ВОПРОСУ ОБ АЛГОРИТМАХ ПРИБЛИЖЕННОГО ВЫЧИСЛЕНИЯ МИНИМУМА ВЫПУКЛОЙ ФУНКЦИИ ПО ЕЕ ЗНАЧЕНИЯМ}

\section{В. Ю. Протасов}

1. Введение. Пусть нам дана вьпуклая функция $f: G \rightarrow \mathbb{R}, f \neq$ const, где $G \subset \mathbb{R}^{n}$ - вьпуклое тело (выпукльй компакт с непустой внутренностью). Мы можем вычислить функцию $f$ в любой данной точке $x \in G$, и это вычисление составляет одну операцию. Требуется найти минимум функции $f$ с заданной точностью, затратив при этом возможно меньшее число операций.

Работа посвящена алгоритму приближенной минимизации вьпуклой функции по ее значениям, которьй затрачивает (асимптотически по размерности области определения) существенно меньшее число операций, чем ранее построенные алгоритмы.

2. Постановка задачи. Пусть $G \subset \mathbb{R}^{n}$ - вьпуклое тело, $V(G)$ - совокупность всех вьпуклых непрерьвных функций на $G$. Для заданного $\varepsilon$ $(1 / 2>\varepsilon>0)$ обозначим через $\nu(G, \varepsilon)$ минимальное число операций, необходимое для того, чтобы для любой функции $f \in V(G)$ найти точку $x \in G$, в которой относительное отклонение от минимума не превосходит $\varepsilon$ (т.е. $\left.\left(f(x)-\min _{t \in G} f(t)\right) /\left(\max _{t \in G} f(t)-\min _{t \in G} f(t)\right) \leqslant \varepsilon\right)$.

Требуется оценить величину $\nu(G, \varepsilon)$ сверху.

Для данной точки $y \in G$ обозначим через $G^{\prime}(y)$ - выпуклое тело, лежащее в $G$ и обладающее следующим свойством:

$$
\forall t \in G \backslash G^{\prime}(y) \quad f(t) \geqslant f(y) .
$$

В работе [1] доказано, что если для данной точки $y \in G$ существует тело $G^{\prime}(y)$ такое, что

$$
\operatorname{Vol}\left(G^{\prime}(y)\right) \leqslant \varepsilon^{n} \operatorname{Vol}(G)
$$

то относительное отклонение от минимума в точке $y$ не превосходит $\varepsilon$.

Задача, таким образом, сводится к нахождению такой точки $y \in G$, для которой существует тело $G^{\prime}(y)$ объема, не превосходящего $\varepsilon^{n} \operatorname{Vol}(G)$.

Такая постановка задачи восходит к работе [2], где в одномерном случае, когда $G=[a, b]$, величина $\nu(G, \varepsilon)$ была вычислена точно (известный метод с числами Фибоначчи). 
В многомерном случае, когда наряду со значением функции разрешается вьчислять и ее градиент (при этом функция предполагается гладкой), были построены эффективные алгоритмы и получены оптимальные оценки (см. [1] и [2]).

Для минимизации функции только по ее значениям в многомерном случае оптимальных оценок пока не получено. В работе [1] было показано, что $\nu(G, \varepsilon) \leqslant C(n) \ln (1 / \varepsilon)$, но коэффициент $C(n)$ экспоненциально рос с размерностью $n$. В работе [3] оценка была улучшена:

$$
\nu(G, \varepsilon) \leqslant C \ln \frac{1}{\varepsilon} n^{7} \ln ^{2}(n+1),
$$

где $C$ - не зависит от размерности $n$. Основной целью настоящей работы является построение алгоритма, приводящего к оценке

$$
\nu(G, \varepsilon) \leqslant C \ln \frac{1}{\varepsilon} n^{2} \ln (n+1) .
$$

3. Описание алгоритма. В нашем алгоритме сочетаются метод центрированных сечений А.Ю. Левина и общий замысел метода работы [3], но сама конструкция существенно иная.

ОПРЕДЕЛЕНИЕ. Конус

$$
K=\left\{x \in \mathbb{R}^{n} \mid \frac{(x-a, b)}{\|x-a\| \cdot\|b\|} \geqslant \cos \varphi, a \in G, \varphi \in\left(0, \frac{\pi}{2}\right)\right\}
$$

(т.е. "прямой шаровой" конус с вершиной $a$, направляющим вектором $b$ и раствором $\varphi)$ назовем $(y, \varphi)$-конусом для некоторой точки $y \in G$, если $\forall x \in G \cap K f(x) \geqslant f(y)$.

Если $K$ есть $(y, \varphi)$-конус для некоторой точки $y \in G$, то вьпуклая оболочка множества $G \backslash K$ является $G^{\prime}(y)$-телом.

Идея метода состоит в том, чтобы последовательно “отрезать" от области определения функции $f$ некоторую часть объема, и, после нужного числа шагов, получить точку $y$ и тело $G^{\prime}(y)$, для которого

$$
\operatorname{Vol}\left(G^{\prime}(y)\right) \leqslant \varepsilon^{n} \operatorname{Vol}(G)
$$

Изложению алгоритма предпошлем два вспомогательных утверждения, второе из которых будет доказано в конце статьи.

Лемма 1 [4, с.130]. Для любого выпуклого тела G существует афинное преобразование $F: \mathbb{R}^{n} \rightarrow \mathbb{R}^{n}$, сохраняющее обгем, такое, что тело $F(G)$ обладает следующим свойством:

(*) Существует два кониентрических шара $Г$ и $\gamma$, причем Г содержит данное тело, а $ү$ содержится в нем, и при этом $R / r=n$, әде $R$ и $r$ - радиусы шаров $Г$ и ү соответственно. 
ЛЕмма 2. Если выпуклое тело $G \subset \mathbb{R}^{n}$ обладает свойством (*), $g$ - его иентр тяжести и $K-$ конус с верииной $a \in G$ и раствором $\varphi$, причем $\cos \varphi \leqslant 1 /\left(8 n^{2}\right),\|a-g\| \leqslant R /\left(4 n^{2}\right)$, то

$$
\operatorname{Vol}(\operatorname{Co}(G \backslash K)) \leqslant \frac{11}{12} \operatorname{Vol}(G)
$$

$(\mathrm{Co}(X)$ - выпуклая оболочка множества $X)$.

Алгоритм. Дано: $G \subset \mathbb{R}^{n}$ - вьпуклое тело, $f: G \rightarrow \mathbb{R}^{n}$ - выпуклая функция, число $\varepsilon>0$. Требуется - построить выпуклое тело $G^{\prime}(y) \subset G$, объем которого не превосходит $\varepsilon^{n} \operatorname{Vol}(G)$ и точку $y \in G$.

Нулевой шаг. Применяем к телу $G$ афинное преобразование $F_{0}$ из леммы 1 . Тело $F_{0}(G)$ обладает свойством $(*)$. Находим центр тяжести $g$ тела $F_{0}(G)$.

После этого полагаем $G_{0}=F_{0}(G), g_{0}=g, f_{0}=f\left(F_{0}^{-1}\right)$.

Пусть проделано $m$ шагов. При этом получилось тело $G_{m}$, обладающее свойством $(*)$, его центр тяжести - точка $g_{m}$ и функция $f_{m}: G_{m} \rightarrow \mathbb{R}^{n}$.

$(m+1)-\check{u}$ шаг. С помощью специальной конструкции в множестве $G_{m}$ строится $\left(g_{m}, \varphi\right)$-конус $K_{m+1}$, вершина и раствор которого удовлетворяют условию леммы 2 . После этого остается положить: $G_{m+1}=F_{m+1}\left(\mathrm{Co}\left(G_{m} \backslash K_{m+1}\right)\right), f_{m+1}=f_{m}\left(F_{m+1}^{-1}\right)$, где $F_{m+1}-$ афинное преобразование из леммы 1 для тела $\mathrm{Co}\left(G_{m} \backslash K_{m+1}\right)$.

Согласно лемме $2 \operatorname{Vol}\left(G_{m+1}\right) \leqslant \frac{11}{12} \operatorname{Vol}\left(G_{m}\right)$, поэтому не более чем за $N=n\left[\log _{11 / 12} \varepsilon+1\right]$ шагов объем тела $G_{N}$ станет меньше $\varepsilon^{n} \operatorname{Vol}(G)$. Множество $G^{\prime}(y)=F_{0}^{-1} \cdot F_{1}^{-1} \cdot \ldots \cdot F_{N}^{-1}\left(G_{N}\right)$ будет искомым телом для точки $y=F_{0}^{-1} \cdot F_{1}^{-1} \cdot \ldots \cdot F_{k}^{-1}\left(g_{k}\right)$, где $g_{k}-$ та из точек $\left\{g_{0}, g_{1}, \ldots, g_{N}\right\}$, в которой значение $f_{k}\left(g_{k}\right)$ наименьшее.

Центральньм моментом является метод построения $\left(g_{m}, \varphi\right)$-конуса. Сложность этого метода (количество операций вычисления функции $f$, необходимых для построения конуса) определяет сложность всего алгоритма в целом.

4. Метод построения $\left(g_{m}, \varphi\right)$-конуса. Дано: $G \subset \mathbb{R}^{n}$ - вьпуклое тело, удовлетворяющее свойству $(*), g$ - его центр тяжести, $R$ - радиус шара $\Gamma$ (см. формулировку леммы 1$), f: G \rightarrow \mathbb{R}$ - выпуклая функция.

Требуется построить $(g, \varphi)$-конус $K$ с вершиной, удаленной от точки $g$, не более чем на $R /\left(4 n^{2}\right)$, и раствором $\varphi=\arccos \left(1 /\left(8 n^{2}\right)\right)$ (согласно лемме 1 данные условия гарантируют отсечение 1/12 объема тела на каждом шаге алгоритма).

Определим следующие константы: $T \geqslant 3$ - наименьшее натуральное число, удовлетворяющее неравенству

$$
\left(\cos \frac{2 \pi}{T}\right)^{n} \geqslant \sin \varphi, \quad \delta=\frac{R}{4 n^{2}}, \quad \delta_{k}=\delta\left(\frac{\sin ^{2} 2 \pi / T}{2}\right)^{k}, \quad k=1,2, \ldots, n-1 .
$$


Первый шаг. Проведем через точку $g$ какую-нибудь двумерную плоскость и построим в ней правильньй $T$-угольник с диаметром $\delta_{1}$, одна из вершин которого - точка $g$.

Найдем три соседние вершины этого $T$-угольника (обозначив их по порядку $-b_{1}, a_{1}, c_{1}$, для которых $f\left(a_{1}\right) \geqslant \max \left\{f\left(b_{1}\right), f\left(c_{1}\right), f(g)\right\}$. В качестве $a_{1}$ годится, например, вершина с наибольшим значением функции.

Положим $\alpha_{1}=\left\{x \in \mathbb{R}^{n} \mid\left\|x-b_{1}\right\|=\left\|x-c_{1}\right\|\right\}-(n-1)$-мерная плоскость в $\mathbb{R}^{n}$ (“серединньй перпендикуляр" к отрезку $\left[b_{1}, c_{1}\right]$ ).

Пусть проделано $m$ шагов. Имеем $(n-m)$-мерную плоскость $\alpha_{m}$, точку $a_{m} \in \alpha_{m}$.

$(m+1)-\tilde{u}$ шаг. Проводим через точку $a_{m}$ двумерную плоскость в $\alpha_{m}$ и построим в ней правильный $T$-угольник с диаметром $\delta_{m+1}$, так, чтобы точка $a_{m}$ была одной из его вершин. Найдем в нем три соседние вершины $b_{m+1}, a_{m+1}, c_{m+1}$, для которых выполняется неравенство $f\left(a_{m+1}\right) \geqslant$ $\max \left\{f\left(b_{m+1}\right), f\left(c_{m+1}\right), f\left(a_{m}\right)\right\}$, и проводим $(n-m-1)$-мерную плоскость $\alpha_{m+1}$ в $\alpha_{m} ; \alpha_{m+1}=\left\{x \in \alpha_{m} \mid\left\|x-b_{m+1}\right\|=\left\|x-c_{m+1}\right\|\right\}$.

После $(n-1)$-го шага получаем прямую $\alpha_{n-1}$ ( "одномерная плоскость") и точку $a_{n-1}$ на ней. Оказьвается, эти прямая и точка будут осью и вершиной соответственно искомого конуса.

ТЕОремА. Конус $K$ с раствором $\varphi=\arccos \left(1 /\left(8 n^{2}\right)\right)$, вершиной $a_{n-1}$ и направляющим вектором $\left(a_{n-1}-b_{n-1}\right)+\left(a_{n-1}-c_{n-1}\right)$ есть

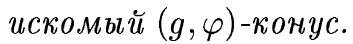

ДокАЗАтЕЛьство. Рассмотрим плоскость $\alpha_{n-m}(2 \leqslant m \leqslant n)$, образующуюся на $(n-m)$-том шаге алгоритма. Пусть $K_{m} \subset \alpha_{n-m}$ есть $m$-мерный конус с направляюшим вектором $\left(a_{n-1}-b_{n-1}\right)+\left(a_{n-1}-c_{n-1}\right)$, вершиной $a_{n-1}$ и раствором $\varphi_{m}=\arcsin \left(\cos ^{m}(2 \pi / T)\right)$.

Докажем по индукции, что $K_{m}$ есть $\left(a_{n-1}, \varphi_{m}\right)$-конус для функции $f$, ограниченной на множестве $G \cap \alpha_{n-m}$.

База $(m=2)$. В этом случае возникает "конус" $K_{2}$ на двумерной плоскости $\alpha_{n-2}$. Надо доказать, что $f\left(a_{n-1}\right)-$ минимум функции $f$ на множестве $K_{2}$. Предположим, что это не так, т.е. что существует точка $x \in K_{2}$, для которой $f(x)<f\left(a_{n-1}\right)$. Тогда из вьпуклости функции $f$ следует, что $f(y)>f\left(a_{n-1}\right)$, где $y$ - точка пересечения прямой $x a_{n-1}$ с отрезком $\left[b_{n-1}, c_{n-1}\right]$. Так как $f\left(a_{n-1}\right) \geqslant \max \left\{f\left(b_{n-1}\right), f\left(c_{n-1}\right)\right\}$, то $f(y)>\max \left\{f\left(b_{n-1}\right), f\left(c_{n-1}\right)\right\}$. Но $y$ является внутренней точкой отрезка $\left[b_{n-1}, c_{n-1}\right]$, поэтому последнее неравенство невозможно для выпуклой функции. Противоречие.

В доказательстве мы использовали тот факт, что конус $K_{2}$ целиком лежит внутри угла, вертикального к углу $b_{n-1} \widehat{a_{n-1}} c_{n-1}$. Это следует из легко доказьваемого неравенства

$$
b_{n-1} \widehat{a_{n-1}} c_{n-1}=\frac{\pi}{2}-\frac{\pi}{T}>\arcsin \left[\left(\cos \frac{2 \pi}{T}\right)^{2}\right]=\varphi_{2} .
$$


Индуктивный переход $m \rightarrow(m+1)$. Имеем $\left(a_{n-1}, \varphi_{m}\right)$-конус $K_{m}$, лежащий в $\alpha_{n-m}$.

Надо доказать, что конус $K_{m+1}$ с вершиной $a_{n-1}$ и раствором $\varphi_{m+1}$ будет $\left(a_{n-1}, \varphi_{m+1}\right)$-конусом, иначе говоря, $\forall x \in K_{m+1} f(x) \geqslant f\left(a_{n-1}\right)$.

Возьмем любую точку $x \in K_{m+1}$. Надо доказать, что $f(x) \geqslant f\left(a_{n-1}\right)$. Так как $x$ лежит внутри конуса, то угол $\widehat{x a_{n-1}} b$ будет меньше угла $\varphi_{m+1}$, где $b \in \alpha_{n-1}$ и $b x \perp a_{n-1} x$. Положим $h=\alpha_{n-m} \cap b_{n-m} x$. Не ограничивая обшности, можно считать, что точки $x$ и $b_{n-m}$ лежат в пространстве $\alpha_{n-m-1}$ по разные стороны относительно гиперплоскости $\alpha_{n-m}$, иначе вместо $b_{n-m}$ берем $c_{n-m}$.

Докажем, что $\widehat{h a_{n-1}} b<\varphi_{m}$. В этом случае точка $h$ будет лежать внутри конуса $K_{m}$ и по предположению индукции $f(h) \geqslant f\left(a_{n-1}\right)$.

Так как $h \in\left[x, b_{n-m}\right]$, то, в силу вьпуклости $f$, имеем неравенство $f(h) \leqslant \max \left\{f(x), f\left(b_{n-m}\right)\right\}$. Наконец, $f\left(b_{n-m}\right) \leqslant f\left(a_{n-m}\right) \leqslant f\left(a_{n-1}\right)$ (из алгоритма построения конуса). Объединяя три неравенства, получим $f(x) \geqslant f\left(a_{n-1}\right)$, что и требуется для индуктивного перехода.

Остается показать, что $\widehat{h a_{n-1}} b<\varphi_{m}$. Введем обозначения: $b^{\prime}-$ проекция точки $b$ на прямую $h a_{n-1}, \beta$ - двумерная плоскость, проходящая через точки $b_{n-1}, a_{n-1}, x$. $H_{b}$ - расстояние от $b$ до плоскости $\beta$. Имеем:

$\sin \left(\widehat{h a_{n-1}} b\right)=\frac{\left\|b b^{\prime}\right\|}{\left\|a_{n-1} b\right\|}=\frac{\left\|b b^{\prime}\right\|}{\|b x\|} \cdot \frac{\|b x\|}{\left\|b a_{n-1}\right\|}<\frac{\left\|b b^{\prime}\right\|}{H_{b}} \sin \varphi_{m+1}=\frac{\sin \varphi_{m+1}}{\sin \tau}$,

где $\tau$ - угол между $\alpha_{n-m}$ и двумерной плоскостью $\beta$. Кроме того, очевидно, что $\tau \geqslant c a_{n-1 b_{n}}-m$, где $c=\frac{1}{2}\left(c_{n-m}+b_{n-m}\right)$,

$$
\begin{aligned}
& \sin \tau \geqslant \sin \left(c a_{n-1 b_{n}}-m\right)=\cos \left(c b_{n-m} a_{n-1}\right) \\
& \geqslant \cos \left(2 c b_{n-m} \widehat{a_{n}}-m\right)=\cos \frac{2 \pi}{T} \text {. }
\end{aligned}
$$

Поясним второе неравенство:

$$
\begin{aligned}
\left\|c a_{n-1}\right\| & \leqslant\left\|c a_{n-m}\right\|+\left\|a_{n-m} a_{n-1}\right\| \\
& \leqslant\left\|c a_{n-1}\right\|+\sum_{i=n-m}^{n-2}\left\|a_{i} a_{i+1}\right\| \leqslant \sum_{i=n-m}^{n-2} \delta_{i} \\
& \leqslant\left\|c a_{n-m}\right\|+\delta_{n-m-1}\left(\frac{\sin ^{2} \pi / T}{2}+\cdots+\left(\frac{\sin ^{2} \pi / T}{2}\right)^{m-1}\right) \\
& \leqslant\left\|c a_{n-m}\right\|+\delta_{n-m-1} \sin \frac{2 \pi}{T} \leqslant 2\left\|c a_{n-m}\right\| .
\end{aligned}
$$

Последнее неравенство следует из соотношений в правильном $T$-угольнике с диаметром $\delta_{n-m-1}$. Итак,

$$
\sin \widehat{h a_{n-1} b}<\frac{\sin \varphi_{m+1}}{\cos (2 \pi / T)}=\left(\cos \frac{2 \pi}{T}\right)^{m},
$$


т.е. $\widehat{h a_{n-1}} b<\varphi_{m}$.

Для завершения доказательства остается положить $m=n, \alpha_{0}=\mathbb{R}^{n}$, $K=K_{n}$. Получаем $n$-мерньй $\left(a_{n-1}, \varphi\right)$-конус $K$ с вершиной $a_{n-1}$ и раствором $\varphi=\arccos \left(1 /\left(8 n^{2}\right)\right)$. В силу выбора точек $a_{1}, \ldots, a_{n-1}$ имеем неравенства $f(g) \leqslant f\left(a_{1}\right) \leqslant \cdots \leqslant f\left(a_{n-1}\right)$. Поэтому всякий $\left(a_{n-1}, \varphi\right)$-конус будет также и $(g, \varphi)$-конусом. В частности, полученньй конус $K$ является $(g, \varphi)$-конусом. Остается оценить расстояние от вершины конуса $K$ до центра тяжести $g$ :

$$
\left\|g a_{n-1}\right\| \leqslant\left\|a_{n-1} a_{n-2}\right\|+\cdots+\left\|a_{2} a_{1}\right\|+\left\|a_{1} g\right\| \leqslant \sum_{k=1}^{n-1} \delta_{k} \leqslant \delta .
$$

Итак, конус $K$ является искомьм $(g, \varphi)$-конусом. Теорема доказана.

5. Оценка сложности алгоритма. При реализации алгоритма мы должны построить $N=n\left[\log _{11 / 12} \varepsilon+1\right]$ конусов. Метод построения $(g, \varphi)$-конуса состоит из $(n-1)$ шага. На $k$-м шаге операция вьчисления функции в точке используется только при нахождении вершины $a_{k+1}$ правильного $T$-угольника, для которой $f\left(a_{k+1}\right) \geqslant \max \left\{f\left(a_{k}\right), f\left(b_{k+1}\right), f\left(c_{k+1}\right)\right\}$, где $b_{k+1}$ и $c_{k+1}-$ соседние с $a_{k+1}$ вершины $T$-угольника, а вершина $a_{k}$ наперед задана. Обозначим количество операций, необходимых для этого, через $\mu(T)$.

Весь алгоритм, таким образом, использует $(n-1) n\left[\log _{11 / 12} \varepsilon+1\right] \mu(T)$ операций. Осталось оценить величину $\mu(T)$.

Лемма 3. $\mu(T) \leqslant C_{1} \ln T$, әде $C_{1}$ - некая константа.

ДокАЗАТЕЛьСТво. Для доказательства этого утверждения предъявим способ нахождения в $k$-м многоугольнике вершин $b_{k+1}, a_{k+1}, c_{k+1}$, для которых $f\left(a_{k+1}\right) \geqslant \max \left\{f\left(a_{k}\right), f\left(b_{k+1}\right), f\left(c_{k+1}\right)\right\}$, затрачивающий не более чем $C_{1} \ln T$ операций вычисления функции $f$.

Пусть $A_{1}, A_{2}, \ldots, A_{T}$ - наш $T$-угольник. Можно считать, что $T=2^{m}$, иначе добавим к нему еще вершины $A_{T+1}, \ldots, A_{2^{m}}\left(2^{m-1}<T \leqslant 2^{m}\right)$, доопределив в них функцию $f$ таким образом, чтобы последовательность $f\left(A_{T}\right), f\left(A_{T+1}\right), \ldots, f\left(A_{2}\right), f\left(A_{1}\right)$ была монотонной.

Способ таков.

Нулевой шаг. Полагаем $A_{1}=a_{k}$. Из вершин $A_{2}, A_{2^{m-2}}, A_{2^{m-1}}$, $A_{3.2}-2$, делящих периметр $2^{m}$-угольника на четыре равные части, выберем вершину с наибольшим значением функции. Обозначим эту вершину через $N_{1}$, а ближайшие к ней (из этих четырех) - через $L_{1}$ и $P_{1}$.

$i$-й шаг. Вычислим $f\left(M_{i}\right), f\left(O_{i}\right)$, где вершины $M_{i}, N_{i}$ и $O_{i}$ делят дугу $L_{i} P_{i}$ на четыре равные части (вершины $L_{i}, N_{i}$ и $P_{i}$, а также значения функции в них вычислялись на предыдущих шагах таким образом, что $\left.f\left(N_{i}\right) \geqslant f\left(L_{i}\right), f\left(N_{i}\right) \geqslant f\left(P_{i}\right)\right)$. Из вершин $N_{i}, M_{i}$ и $O_{i}$ выберем вершину 
с максимальньм значением функции, обозначим ее через $N_{i+1}$ и ближайшие к ней (из этих пяти) - через $L_{i+1}$ и $P_{i+1}$. Затем переходим к $(i+1)$-му шагу.

После $(m-1)$ шага полагаем $a_{k+1}=N_{m}, b_{k+1}=L_{m}, c_{k+1}=P_{m}$, и искомые вершины найдены. При этом функция вычислялась $2 m$ раз.

$$
2 m \leqslant 2 \log _{2} T+1 \leqslant C_{1} \ln T,
$$

где $C_{1}-$ константа.

Лемма 3 доказана.

Из определения числа $T$ :

$$
\left(\cos \frac{2 \pi}{T}\right)^{n} \geqslant \sin \left(\arccos \frac{1}{8 n^{2}}\right),
$$

легко получить оценку

$$
\ln T \leqslant C_{2} \ln (n+1) .
$$

Итак, получаем

$$
\mu(T) \leqslant C_{3} \ln (n+1),
$$

$C_{3}$ - некая константа.

Итоговая оценка сложности алгоритма такова: общее количество операций не превосходит

$$
(n-1) n\left[\log _{11 / 12} \varepsilon+1\right] C_{3} \ln (n+1),
$$

$C_{3}$ - константа, не зависящая от $n$ и $\varepsilon$.

Алгоритм, таким образом, привел нас к оценке

$$
\nu(G, \varepsilon) \leqslant C \ln \frac{1}{\varepsilon} n^{2} \ln (n+1),
$$

$C$ - константа.

В заключение приводим

ДоКАЗАТЕЛЬСТВо ЛЕммы 2. Обозначим через $c$-направляюший вектор конуса $K$. Положим

$$
t_{0}=\frac{R}{\|c\| 4 n^{2}}, \quad b=a+t_{0} c .
$$

Две гиперплоскости, проходящие через точки $g$ и $b$, и перпендикулярные вектору $c$ (расстояние $h$ между ними, очевидно, не превосходит $R /\left(2 n^{2}\right)$ ), делят тело $G$ на три части $G^{1}, G^{2}, G^{3}$ соответственно. Тела $G^{3}$ и $\operatorname{Co}(G \backslash K)$ не пересекаются.В самом деле, если $x$ - какая-нибудь точка из $G^{3}$, то косинус угла между векторами $c$ и $(x-a)$ не меньше, чем

$$
\frac{\|a b\|}{\|a x\|} \geqslant \frac{R}{4 n^{2}} \cdot \frac{1}{2 R}=\frac{1}{8 n^{2}}
$$


и, следовательно, $x \in K$. Но из выпуклости множества $G^{1} \cup G^{2}$ следует, что $\operatorname{Co}(G \backslash K) \subset G^{1} \cup G^{2}$, а, значит, $G^{3} \cap \operatorname{Co}(G \backslash K)=\varnothing$.

Так как часть $G^{1}$ отсекается от $G$ гиперплоскостью, проходящей через центр тяжести тела $G, \operatorname{to} \operatorname{Vol}\left(G^{1}\right) \leqslant \operatorname{Vol}(G)(1-1 / e)\left(\right.$ см. [5]). $\operatorname{Vol}\left(G^{2}\right)$ запишем в виде интеграла $\int_{0}^{h} S(t) d t$, где $S(t)-(n-1)$-мерньй объем сечения тела $G$ гиперплоскостью, проходящей через точку $g+t(c /\|c\|)$ перпендикулярно вектору $c$.

По теореме о среднем $\exists t_{1} \in[0, h] \int_{0}^{h} S(t) d t=h S\left(t_{1}\right)$. Так как объем сечения не превосходит отношения величины $n \operatorname{Vol}(G)$ к проекции $G$ на нормаль к плоскости сечения, а эта проекция, в свою очередь, не меньше $2 r$, то

$$
S\left(t_{1}\right) h \leqslant \frac{n \operatorname{Vol}(G)}{2 r} h \leqslant \frac{n \operatorname{Vol}(G)}{2 r} \cdot \frac{R}{2 n^{2}}=\operatorname{Vol}(G) \frac{R}{4 n r} \leqslant \frac{1}{4} \operatorname{Vol}(G) .
$$

В итоге получаем,

$$
\begin{aligned}
\operatorname{Vol}(\operatorname{Co}(G \backslash K)) & \leqslant \operatorname{Vol}\left(G^{1}\right)+\operatorname{Vol}\left(G^{2}\right) \leqslant \operatorname{Vol}(G)\left(1-\frac{1}{e}\right)+\frac{1}{4} \operatorname{Vol}(G) \\
& \leqslant \frac{11}{12} \operatorname{Vol}(G),
\end{aligned}
$$

что и требовалось.

Лемма 2 доказана.

Московский государственный университет

Поступило

им. М.В. Ломоносова

18.04 .94

\section{СПИСОК ЦИТИРОВАННОЙ ЛИТЕРАТУРЫ}

[1] Немировский А. С., Юдин Д. Б. Информативная сложность и эффективные методы решения выпуклых экстремальных задач // Экономика и матем. методы. 1976. Т. 12. № 2. С. 357-369.

[2] Левин А. Ю. Об одном алгоритме минимизации вьпуклых функций // Докл. AH CCCP. 1965. T. 160. №6. C. 1241-1243.

[3] Юдин Д. Б., Горяшко Д. М., Немировский А. С. Математические методы оптимизации устройств и алгоритмов на АСУ. М.: Радио и связь, 1982.

[4] Гусман М. Дифференцирование интегралов в $\mathbb{R}^{n}$. М.: Мир, 1978.

[5] Митягин Б. С. Два неравенства для объемов выпуклых тел // Матем. заметки. 1959. Т. 5. № 5. С. 20-26. 